\title{
Getting lost? Touch and you will find! The user-centered design process of a touch screen
}

\author{
Bieke Zaman, Rogier Vermaut \\ CUO/IBBT - K.U.Leuven, E. Van Evenstraat 2A, 3000 Leuven, Belgium \\ Bieke.Zaman@soc.kuleuven.be, Rogier.vermaut@soc.kuleuven.be \\ Phone (+32) $16323251,(+32) 16323653$
}

\begin{abstract}
Recent reforms in office concepts have led to new intensification strategies that aim at more flexibility and efficiency. Hot desking is one of these new working practices that reduces office space per worker. These new office concepts however, pose new challenges and problems to solve. This paper describes the development phase of an innovative touch screen application for location based services to overcome the problematic edge effects of hot desking such as missing workspace awareness and poor communication. We followed a user-centered design (UCD) process to develop and test the interface so that it could be gradually modified and tailored to the demands and expectations of the end users. First, a methodological overview of the different phases of the UCDprocess is given. Then, the results of each phase are discussed, focusing on several interface elements. Finally, the most important and striking results are summarized.
\end{abstract}

Key Words: Touch screen interface, hot desking, user-centered design process, usability testing, contextual inquiry, conceptual model, paper prototyping

\section{Introduction}

Recent developments in office environments can be classified into two major trends. On the one hand, team work becomes more and more important as an organizational condition. On the other hand, more attention is being paid to the reorganization of office concepts to focus on higher personal mobility [1]. In this last trend towards more personal mobility and together with the business' strive for more flexibility and efficiency, new office concepts arose.

Hot desking is one of the more recent office intensification strategies aiming to reduce office space per worker [2]. Hot desking is a new working practice that offers a compensation for space needs in companies that focus on the job activities rather than on the individuals themselves. People then work together based on their job responsibilities without the prerequisite of a shared office environment. In practice, employees can choose an available place instead of going to the same desk every morning $[2,3]$.

Although hot desking has many advantages in terms of flexibility and efficiency, problems such as missing workspace awareness, lacking group cohesion and poor 
communication must be dealt with [1]. That is why computer supported cooperative work (CSCW) becomes very important [3]. In this paper, however, we focus on the consequences and edge effects of hot desking apart from the conditions for CSCW. When employees do not have a place of their own, some uncertainties turn up. For example, how does one know whether someone is at his office, on holiday, on the road or working at home? How to find someone's exact working place when face-toface contact is necessary? How to locate someone if one cannot reach him on his mobile? As a result of these and similar questions, the go-ahead was given for the this project. The Belgian telecom company Alcatel-Lucent recently chose to switch to hot desking. Alcatel-Lucent is part of the worldwide Alcatel group and focuses on the development of broadband technologies and related applications. To overcome hot desking problems, Alcatel-Lucent decided to invest in a new technological solution that should allow people to easily locate others. The aim of this paper is to describe the user-centered design process rather than the technological development phases.

From the beginning, the project aimed at developing both a touch screen as well as a PC-based solution to overcome workspace awareness related problems in AlcatelLucent's hot desking environment. This objective poses a double challenge. Since a PC graphical user interface (GUI) cannot simply be turned into a touch screen GUI, we had to find the right balance between consistency and platform specific requirements. Contrary to a desktop computer GUI, touch screens are mostly used in public environments and characterized with hurried (and often novice) users, easy (and often wizard-like) interfaces and intuitive interaction forms [4].

This paper will focus on the user-centered design process of the touch screen application. The paper begins with a description of the methodology of the UCD process. Then, we discuss the main results according to the different phases of the design process. In particular, we focus on the results of respectively the contextual inquiry, the conceptual model, the paper prototyping and the iterative usability testing. Finally, the paper concludes with a summary of the most important and striking results.

\section{Methodology}

To develop a user friendly touch screen application, we followed a user-centered design process. This process involves the user in each phase of the design chain. This way, the end-product is geared as much as possible to the wishes and characteristics of the users, resulting in a high level costumer satisfaction [5].

The first phase of the UCD process consisted of a contextual inquiry. In this phase, we not only tried to comprehend who the main users of the final application are, but also how these users perform their present as well as their future tasks [6].

The next phase entailed the drawing of a conceptual model. The conceptual model is the basis of the user interface. It is a first abstract design in which the information gathered during the contextual inquiry has been processed. This way, the conceptual model leans as much as possible on the way users think about the application [7]. Decisions concerning navigation and interaction are made without concentrating too much on details. In other words, this phase focuses not on design, but on general structure, organization and navigation [8]. 
The conceptual design takes further shape in a number of paper prototypes. Paper prototyping or low-fi prototyping is an excellent way of getting feedback in an early phase of the design process. Putting through adjustments in a later phase of the project would be a more time- and money-consuming matter. On top, paper prototyping gives developers the opportunity to test alternative design ideas before investing in advanced prototypes $[8,9]$.

The next phase comprised the technical development of the interface. As soon as a significant part of the application was developed, we set up a series of usability tests. These tests, rather short and straightforward, goaled the improvement of the development and design process. Finally, a final series of tests took place. In this test, the interface was evaluated thoroughly and in great detail.

\section{Results}

In this section, we amplify on the results of each phase of the UCD process. From the paper prototype phase onwards, we will not describe every little aspect of the interface. In contrast, we examine the origin and evolution of four crucial interface elements: the auto completion window, the soft keyboard, the status of the contact persons and the possibility to ask for information about the colleagues of a contact person.

\subsection{Contextual inquiry}

During our contextual inquiry, we focused on the end users of the system as well as on the tasks they perform in their natural environment. With the contextual inquiry, we wanted to identify all possible requirements end-users need when using the touch screen application. The target group consisted of four types of users: employees, porters, external visitors and secretaries. In this paper however we only focus on the employees since they make the main target group. More particularly, 16 employees were interviewed, concentrating on the following research questions:

- What information do employees need when looking for a colleague in the AlcatelLucent building?

- What difficulties do they encounter when locating and contacting their colleagues?

- What information do they expect to find on the touch screen application?

As we expected, our research findings show the importance of surname as a search term. In second place comes searching on first name. Furthermore, we identified department, name of the colleagues' main project and nickname as possibly important search criteria. During our observations, we found that the employees needed the following information when searching a colleague: information about the presence of the colleagues, detailed information about the colleagues' location, the mobile and fixed telephone number, the email address as well as information about team members. The latter becomes particularly important when the person cannot be found or contacted.

Next, we asked the respondents how they would represent the location of a colleague on a touch screen. A great majority thinks a map is appropriate to visualize the 
location. Suggested representation icons were: a dot, a picture, an arrow or a small person indicating the status of the person, similar to Msn Messenger. These findings confirm the finding of Weilenmann and Leuchovius: "location based services make use of a map metaphor, presenting geographical location in the form of [...] a dot on the map" [10].

\subsection{Conceptual model}

The actual design process of the touch screen application started with the phase of the conceptual model. A conceptual model describes or shows the reasoning of the user based on the information gained during the contextual inquiry.

However, before we could start with the design, we needed to take several considerations into account:

- Touch screens can be manipulated directly while they do not ask for much user experience. No extra input device is necessary for interaction. To a certain degree, this fosters a fast navigation $[4,11]$.

- Sears [12] reports consistent differences between the location users want to touch and where they actually touch. Fingers which are being used instead of the mouse cursor, have a certain size. Therefore, screen elements have to be designed large enough [11]. More specifically, buttons should have a minimum size of $2 * 2 \mathrm{~cm}$ (or $3 / 4$ by $3 / 4$ inch). They should be at least $3 \mathrm{~mm}$ (or $1 / 8$ inch) apart for finger-operated touch screens [13].

- Input is sequential because just one finger is being used. This slows input down compared to normal keyboard input [13]. On top, badly designed interfaces with respect to hand movement can make arm fatigue a major problem [4]. This is why the amount of information to be put in should be limited.

With these and other design considerations kept in mind, we started with the development of the basic application screens. In the following paragraphs, we give a short description of the three main parts of the application: title, search and results page.

The title screen offers two main options: search for a person and search for a room. Although our main target group consists of Dutch speaking employees, we opted for English as the application's default language since all communication is done in English at Alcatel-Lucent. Yet, users can choose the Dutch or French language on the title page.

Users can search a person by name, department, function, name of the project and nickname. At this stage of the project, we could only lean on the lessons learned from our contextual inquiry. Users can give in text with a soft keyboard. As soon as the user enters the first characters of a name, the application shows all possible matches in an auto completion window. It is crucial that the application recognizes all combinations of surname and first name.

The conceptual model for the results page consists of five different building blocks:

- Personal details such as picture, name, phone number, mobile number, email, function and department. 
- The status of the person: a person's status can be 'available', 'busy' or 'not present'. Each status is linked with a color code. Green code means available, grey code means not present, red code means busy.

- A textual description of the location of the person: where can this person be found in the building (building, floor and room)?

- A map of the floor which indicates the route to the location of the contact person starting from the user's position. The person is represented by a small dot that corresponds with its status. The map also indicates some reference points such as restrooms, kitchen, elevators, etc.

- Reachability of the contact person: a textual description of the route to the contact person, information concerning the reachability by phone and a link that loads a list of colleagues.

\subsection{Paper prototyping}

The conceptual model took shape in a few paper prototypes, made up in Microsoft Visio. Paper prototyping makes it possible to experiment with different ideas, thus promoting rapid iterative development. By testing the paper prototypes, we tried to answer the following questions:

- Is the functionality of the interface sufficiently clear, complete and intuitive for the user?

- Are navigation and interaction sufficiently clear?

To answer the questions, we drafted seven tasks. Ten subjects were told to imagine they were entering a large building where they had to meet a project partner. Tasks were formulated as follows: "You are looking for the Director Marketing \& Communication. How would you find him?" or "John Johnson is on his annual holiday. You do not want to disturb him and therefore decide to make an appointment with his secretary. Where can you find her?".

While completing these tasks, we asked each subject to say out loud what they were thinking. Using this think-aloud method, the participants communicated what they did, thought and felt while using the application. This made it possible to evaluate the interface thoroughly and in great detail. Pioneering work with the think-aloud protocol shows it to be an effective usability method, capable of identifying serious usability problems with only a few participants [14]. The protocol is very useful in gathering qualitative data about users' mental models, as well as their general impressions and feelings about navigation and design [15].

In the following sections, we discuss our major findings concerning four crucial interface elements.

\subsubsection{Auto completion}

We already formulated the expectation that the system should be able to recognize all searches that combine first name and surname. This assumption was confirmed by the user tests. Subjects entered all different combinations: mostly first name + surname, but also surname + first name and even only surname or first name (see figure $1)$. 


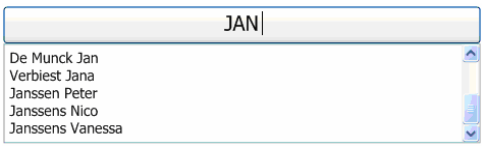

Fig. 1. Auto completion: while typing, the system suggests all possible matches.

\subsubsection{Keyboard}

A study of Mackenzie, Zhang and Soukoreff [16] suggests that a Qwerty keyboard is convenient in situations where users must get started fast and easy. Since the application will be most often used by novices, we chose to offer a Qwerty keyboard by default.

Participants did not seem to have significant difficulties using the Qwerty keyboard. However, most users did notice making use of a Qwerty keyboard. This because in Flanders, an Azerty keyboard is more widespread than a Qwerty keyboard. One out of 16 subjects explicitly said he preferred an Azerty-keyboard. Based on the users' remarks, we replaced the default Qwerty keyboard with an Azerty keyboard. Nevertheless, we decided to offer the possibility to switch from the default Azerty keyboard to a Qwerty or alphabetical keyboard. In the previous version (see figure 2, left), the keys were also too close to each other and too small to select. That is why we enlarged and separated the keys significantly in the improved version (see figure 2 , right). Finally, we removed the 'done button', which caused too much confusion in combination with the search button.
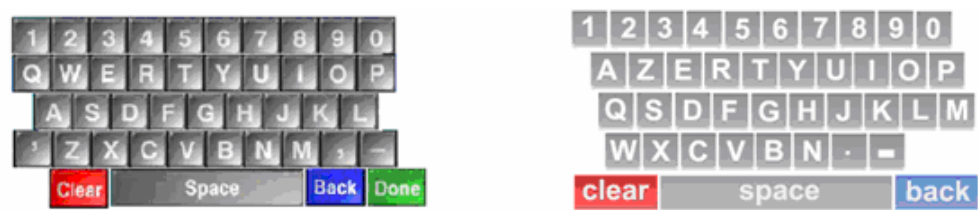

Fig. 2. The keyboard on the left-hand side caused problems and was therefore replaced by a more user friendly keyboard, here placed at the right-hand side of the above figure.

\subsubsection{Status}

Most participants showed an intuitive understanding of the meaning of the status 'available' and 'not present'. However, subjects showed a various understanding of the status 'busy'. Whereas some subjects preferred not to contact a busy colleague, others still tried to call this person. Their reasoning was as follows: "I would contact this person anyway. After all, I have an appointment and need to speak to him urgently". The different interpretations made us decide to elaborate two different types of 'busy' colleagues:

- A status 'do not disturb' which indicates that the person cannot be disturbed (because he is in meeting for example).

- A status 'busy' where a person prefers not to be contacted but it is allowed by exception. 


\subsubsection{Contact colleagues}

During the tests, we realized that users could only consult the list of colleagues when they clicked on 'show map' (see figure 3, left). However, users already needed this option as soon as they noticed that a person was not present or busy. Thus, we improved the interface by offering the button 'show colleagues' already at this screen (see figure 3, right). The location related information was offered in a separate window.
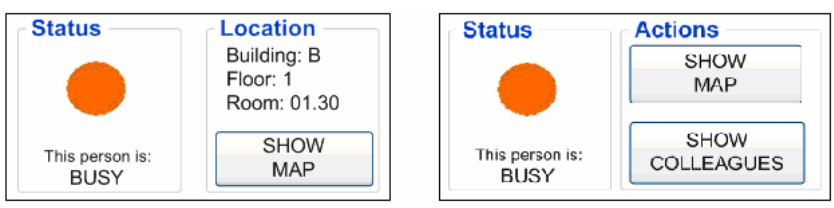

Fig. 3. The interface on the left-hand side did not have a direct link to the action 'show colleagues'; this was improved as shown on the right-hand side of the above figure.

\subsection{Second series of usability tests}

In contrast to the first usability test sessions that made us develop and test different low-fi paper prototypes, the second and third series of usability tests were performed using a working hi-fi prototype. The second series of tests targeted extra guidelines for developing the touch screen application.

We drew up five tasks for ten participants. Each test took about half an hour. We held these test on location and used a portable usability lab. The portable usability lab is suitable for field research, where the usability of the application in question can be researched in the natural (work) environment of the user. In the following section, the main findings of the second series of usability tests are discussed.

\subsubsection{Auto completion}

The positioning of the auto completion window was problematic. The auto completion window partly covered the keyboard. As a consequence, all users got frustrated because they could not strike some of the keyboard characters. Also, participants suggested capitalising the first letter of the names in the auto completion list. As a consequence the window was repositioned and the first letters were capitalised.

\subsubsection{Keyboard}

Our modifications concerning the keyboard were tested in the second series of usability tests. The buttons to change the type of keyboard proved not user friendly. Buttons were in the left bottom corner whereas the keyboard is situated in the right corner. Also, three options were offered while only two are needed. If, for example, the Azerty keyboard is selected, one should only have a choice between the 'Qwerty' and 'alphabetic' keyboard. 


\subsubsection{Status}

The difference between the busy status and the do not disturb status was immediately clear to our participants. Yet, the majority of our participants would first call an available contact person before visiting him/her. This made us conclude that a call option would be of great value for the application. Therefore, we changed the status icons and integrated a call button (see figure 4). When pressing this button, the fixed number of the contact person is dialled automatically via Voice over IP. This call button was repeatedly suggested and highly appreciated by the majority of our test users during all test sessions.

\subsubsection{Contact colleagues}

The repositioning of the 'show colleagues' button proved very successful. However, the findings of both usability tests show that users distinguish between secretary and other colleagues. The majority of test users suggested adding a new button 'show secretary'. As a consequence, we added the button show secretary at the page with the map (see figure 4).

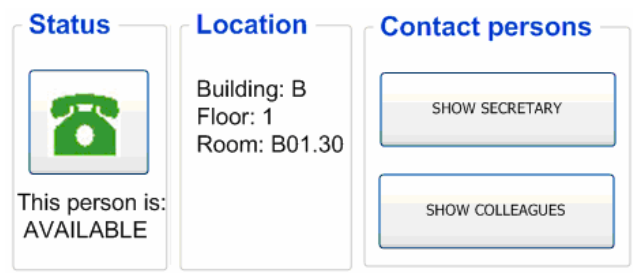

Fig. 4. The buttons 'show secretary' and 'show colleagues' were added at the individual page.

\subsection{Third series of usability tests}

In our final usability test session, we concentrated on the modifications that were implemented following the second series of tests. Of course, we also paid attention to any other usability problems that persisted.

\subsubsection{Auto completion}

The problems with the positioning of the auto completion window were corrected. However, few participants noticed that the names in the auto completion list were ordered randomly whereas they expected an alphabetic list. As a result, we took this consideration into account.

\subsubsection{Keyboard}

The problems with the keyboard selection were solved completely. Users who wanted to change the type of keyboard did so easily. Our test participants also expected a clear-button next to the search input box. We followed this recommendation. This way we also avoided arm fatigue [4] by limiting the distance between the clear button and the search input box. 


\subsubsection{Status}

In this final test, a new problem with the status 'not present' surfaced. When a person is not present, users can still press the button 'show map'. To our test persons, this was rather strange since "a person that is not present, can't be shown on a map". Consequently, in this case we decided to replace the button 'show map' with 'show secretary'. Users whose contact person turns out to be absent, can now address very easily to the secretary or one of the colleagues.

\subsubsection{Contact colleagues}

Since we moved the button 'show colleagues' after the first tests, the functionality of the part 'How to reach?' was outstripped. Because this building block only shows information that already can be found at the top of the page, namely the phone numbers, the email address and the link to the colleagues, we decided to drop this building block altogether.

\section{Conclusion}

The present study examined the user-centred design (UCD) process of a touch screen interface directed at locating colleagues or contact persons in a hot desking environment. Modifications to all designs were based on the feedback of these users.

During the UCD process, some important improvements were made with regard to the developed touch screen. Summarizing, it may be stated that there are six major elements that guarantee a user friendly and successful touch screen application for location based services. First, status information is crucial for an application that locates persons. People with a certain level of computer experience are familiar with different representations of someone's status (like e.g. use of colours or icons). The results of our study show that a single status 'busy' is too ambiguous. That is why one should make the distinction between 'busy' -which means that someone can still be disturbed- and 'do not disturb' -which means that someone is really busy and therefore cannot be interrupted. Secondly, a map is the most obvious way to represent a person's location. This map should be presented from the users' point of view and show the most important reference points such as the elevator, the stairs, the toilets and the restaurant. Our results prove that a function 'show colleagues' is needed when a person cannot be found or contacted. In that case, a distinction between secretary and colleagues should be made. As for the touch screen keyboard, one should make sure buttons are very large (minimum $2 * 2 \mathrm{~cm}$ ) and well separated. The type of keyboard (Azerty, qwerty or alphabetic) is of a lower priority. Further, our results prove that auto completion is a handy and efficient feature that facilitates search queries. Last but not least, the results make clear that a phone service integrated in the application via VOIP is the killer application that guarantees a successful functionality. 
Acknowledgements. This paper is the result of research carried out as part of the Tcase project, funded by the Interdisciplinary institute for Broad Band Technology (IBBT). IBBT is a research institute founded by the Flemish Government, that brings together commercial companies (like in this project Alcatel-Lucent) with academic research groups of different backgrounds (i.c. CUO for user-centered design and IBCN for broadband communication networks).

\section{References}

1. Röcker, C., Prante, T., Streitz, N.A., van Alphen, D.: Using Ambient Displays and Smart Artefacts to Support Community Interaction in Distributed Teams. In: Proceedings of the OZCHI Conference 2004, University of Wollongong, Australia

2. Lizieri, C.M.: Occupier Requirements in Commercial Real Estate Markets. In: Urban Studies, vol. 40, issue 5/6, (2003) 1151-1169

3. Luff, P., Health, C.: Mobility in collaboration. In: Proceedings of the 1998 ACM conference on Computer supported cooperative work, ACM Press New York (1998) 305-314

4. Sjöberg, S.: A Touch Screen Interface for Point-Of-Sale Applications in Retail Stores. Master's Thesis in Computing Science, Umea University, Sweden (2006)

5. Vredenburg, K., Isensee S., Righi, C.: User Centered Design. An integrated approach. PTR Prentice Hall, Indianapolis (2002)

6. Beyer, H., Holtzblatt, K.: Contextual design: Defining customer-centered systems. Morgan Kaufmann Publishers, San Francisco (1998)

7. Benyon, D., Turner, P., Turner, S.: Designing interactive systems: People, Activities, Contexts, Technologies. Addison Wesley, Boston (2004)

8. Stone, D., Jarrett, C., Woodroffe, M., Minocha, S.: User Interface Design and Evaluation. Morgan Kauffman Publishers, San Francisco (2005)

9. Snyder, C.: Paper prototyping. The fast and easy way to design and refine user interfaces. Morgan Kaufmann Publishers, San Francisco (2003)

10. Weilenmann, A., Leuchovius, P.: "I'm waiting where we met last time": exploring everyday positioning practices to inform design. In: Proceedings of the third Nordic conference on Human-computer interaction, ACM Press New York (2004) 33-42

11. Albinsson, P.A., Zhai, S.: High precision touch screen interaction. In: Proceedings of the SIGCHI conference on Human factors in computing systems, ACM Press New York (2003) $105-112$

12. Sears, A.: Improving Touch Screen Keyboards: design issues and a comparison with other devices. In: Interacting with computers, Vol. 3. Elsevier, New York (1991) 253-269

13. Waloszek, G.: Interaction Design Guide for Touch screen Applications, SAP Design Guild (2000). Found on: http://www.sapdesignguild.org/resources/TSDesignGL/INDEX.HTM

14. Nielsen, J.: Evaluating the Thinking Aloud Technique for Use by Computer Scientists. In: Advances in Human-Computer Interaction, Vol. 3. Ablex Publishing Corp, Norwood (1993) 69-82

15. Reeves, T.C., Apedoe, X., Hee Woo, Y.H.: Evaluating Digital Libraries: A User-Friendly Guide, National Science Digital Library, University Corporation for Atmospheric Research, 2003. Found on: http://dlist.sir.arizona.edu/398

16. MacKenzie, I. S., Zhang, S. X., Soukoreff, R. W.: Text entry using soft keyboards. Behaviour \& Information Technology, Vol. 18. Taylor \& Francis, Oxfordshire (1999) 235-244 